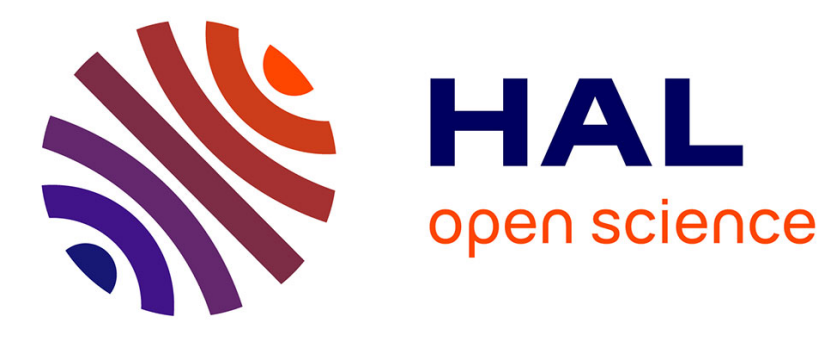

\title{
Conical intersection properties unraveled by the position spread tensor
}

\author{
Alekos Segalina, Antonio Francés-Monerris, Mariachiara Pastore, Thierry \\ Leininger, Stefano Evangelisti, Antonio Monari
}

\section{To cite this version:}

Alekos Segalina, Antonio Francés-Monerris, Mariachiara Pastore, Thierry Leininger, Stefano Evangelisti, et al.. Conical intersection properties unraveled by the position spread tensor. Theoretical Chemistry Accounts: Theory, Computation, and Modeling, 2018, 137 (12), pp.163. 10.1007/s00214018-2377-y . hal-02395774

\section{HAL Id: hal-02395774 \\ https://hal.science/hal-02395774}

Submitted on 24 Nov 2021

HAL is a multi-disciplinary open access archive for the deposit and dissemination of scientific research documents, whether they are published or not. The documents may come from teaching and research institutions in France or abroad, or from public or private research centers.
L'archive ouverte pluridisciplinaire HAL, est destinée au dépôt et à la diffusion de documents scientifiques de niveau recherche, publiés ou non, émanant des établissements d'enseignement et de recherche français ou étrangers, des laboratoires publics ou privés. 


\title{
Conical intersection properties unraveled by the position spread tensor
}

\author{
Alekos Segalina*, Antonio \\ Francés-Monerris*, Mariachiara Pastore, \\ Thierry Leininger, Stefano Evangelisti, \\ and Antonio Monari
}

Received: date / Accepted: date

\begin{abstract}
We explore the application of the electron position spread tensor, i.e. a quantitative measure of the electron delocalization and mobility, to the conical intersection regions of three relevant compounds showing either photoisomerization or chemiluminescence properties. The electronic structure of the involved states has been solved using the complete active space self-consistent field method and the position spread tensor has been computed at the same level of theory. In particular, we show that the total position spread tensor is degenerate between the ground and the excited states, because of the inversion of the electronic nature of the states happening at the crossing areas. We also show that the ground-state position spread tensor shows a discontinuity that may be used to locate conical intersections without the need to explicitly compute the excited state wavefunction. Furthermore, we also report that the spin partition position spread tensor shows a peculiar behavior presenting values close to zero in two of its principal components. We associate those small values to the degeneracy lifting coordinates and hence to the conical intersection branching space.
\end{abstract}

Keywords Conical intersections - position spread tensor - CASSCF . branching space · photophysics and photochemistry

Alekos Segalina, Antonio Francés-Monerris, Mariachiara Pastore, Antonio Monari Université de Lorraine and CNRS, LPCT UMR 7019, F-54000 Nancy, France

E-mail: antonio.monari@univ-lorraine.fr

Thierry Leininger, Stefano Evangelisti

Université Paul Sabatier Toulouse III, LCPQ, Toulouse, France

E-mail: stefano.lcpq@gmail.com

*Those two authors contributed equally 


\section{Introduction}

Conical intersections (CIs) are defined as areas in which the potential energy surfaces (PESs) of two different electronic states of the same spin multiplicity cross. $[1-5]$ As a consequence, they clearly represent regions of the phase space in which the Born-Oppenheimer approximation does not hold anymore, and hence give raise to important non-adiabatic phenomena named internal conversions. In particular, the role of CIs in photochemistry is nowadays recognized as extremely crucial since, because of the high coupling between the crossing PESs, they mediate the non-radiative population transfer between the different electronic states involved in the photoresponse.[6]

The $S_{1} / S_{0}$ crossings are especially relevant because they mediate the ground state recovery of photoexcited molecules. Accessible $S_{1} / S_{0}$ CIs during the excited-state dynamics are related, in general, to non-radiative and relatively fast excited-state decays. In these cases, it is fairly well understood that the CI topology is conditioning the quantum yields and the competition between photostability and photoreactivity. Well-known examples of photostable systems are the DNA canonical nucleobases, $[7,8]$ whereas the retinal molecule embedded in rhodopsin protein $[9,10]$ or the photochemistry of azobenzene[11-13] represent typical examples of photoreactivity. On the other hand, the presence of energy barriers to access the CI regions increases excited-state lifetimes[14, 15] and constitutes an intrinsic characteristic of fluorescent molecules. In the latter, the systems are trapped in one or several excited-state minima for long time periods enabling the ground-state recovery by photon emission. Two examples of luminescent systems that ilustrate these processes are a recently reported family of polyhedral boranes[16,17] and the indole chromophore.[18] Finally, it is noteworthy to mention that CIs also mediate bioluminescence and chemiluminescence phenomena, in which the excited state is thermally populated from the ground state. $[19,20]$ These phenomena are of great importance in nature, and are also intensively exploited by man-made technology especially for the design of analytical methods and biosensors. Further reading about the paramount relevance of CIs in modern theoretical photochemistry can be found in extensive reviews documented elsewhere.[1-5]

Indeed CI regions do not held the same dimensionality as the PES. In fact, in the case of a common molecular system characterized by $M=3 N-6$ vibrational degrees of freedom, where $N$ is the number of nuclei, there are two coordinates, i.e. normal modes, that break the degeneracy between the $\mathrm{PES}$, as a consequence the CIs surface will have a $M-2$ dimensionality.[21] As a corollary, if one considers a diatomic molecule it turns out that the PES can never cross, hence in this case one should talk about avoided crossing regions.[22] On the other hand, for a triatomic system, having 3 vibrational degrees of freedom, the CI area will be reduced to a single degeneracy point characterized by the intersection of two cone-like surfaces, giving raise to the paradigmatic situation that has provided CIs names.

The two degeneracy-breaking coordinates, which together define the socalled $g-h$ branching space, [23] represent a fundamental properties to char- 
acterize the nature and the topology of the CIs. In particular one can usually distinguish between the so-called peaked and sloped topologies. Indeed, while the former induce photo-reactivity, the latter are known to favor photostability instead. The two coordinates defining the branching space can in turn be related to the gradient difference $g$ and the derivative coupling $h$, respectively

$$
\begin{aligned}
& g=\frac{\partial\left(E_{0}-E_{1}\right)}{\partial R} \\
& h=\frac{\partial\left\langle\Psi_{0}|\hat{V}| \Psi_{1}\right\rangle}{\partial R}
\end{aligned}
$$

where $E$ is the energy of the state, $\Psi$ its wavefunction, $\hat{V}$ the coupling hamiltonian element, and $R$ a spatial coordinate. Note that, while the $g$ vectors represent the direction through which the energy difference between the two states increases more rapidly, the $h$ vector describes the direction reducing the coupling.

Since the CIs define a multidimensional surface it is in general necessary to find the minimum energy CI point to properly characterize the excited-state behavior of a given molecular system. This problem, that can be related to a minimization under constraints, has been addressed by different authors in the past and different algorithms exist to provide the minimum energy point of a CI, usually requiring to solve the Schrödinger equation for both the ground and the excited states. [23-29]

In the past, some of us have implemented at ab-initio quantum level the calculation of the so called total position spread (TPS) tensor.[30-35] As it will be detailed in the Methodology section, the TPS is a tensor basically describing the electronic spread and mobility along a given coordinate.[36-39] Originally derived from the modern theory of conductibility, the TPS has been used to most notably characterize the metal-to-insulator transition in model systems, and it has been shown to be able to catch the physical processes related to different kind of transitions such as Mott [30] or Peierls [31] based. Its relationship with the conductibility from the one side, and with the molecular polarizabilities, on the other side, have also been shown both theoretically $[36,39]$ and computationally [30]. As the polarizability, the TPS is strictly a property of a given state of a molecular system but can be expressed as a sum over states formalism. Its calculation has been firstly implemented at Full Configuration Interaction (FCI) level[40,41] to be afterwards extended to the Complete Active Space Self Consistent Field (CASSCF) method.[42] In addition to the total position spread, one can also consider the spin partitioned position spread (SPPS) that explicitly separate the contribution of the same spin and different spin electrons. Its application to the case of simple diatomic molecules has allowed to underline crucial differences in the case of ionic and covalent bonds.

Quite recently, the TPS calculation has been extended from the ground to the electronic excited states and it has unraveled interesting properties in the vicinity of the avoided crossing regions, such as an increase of electron 
mobility.[40,41] In this contribution we plan to extend the application of the TPS and SPPS calculations to the case of CIs obtained for three paradigmatic molecules (Figure 1): i) azobenzene (AB), ii) a simple model of the retinal switch (PSB3), iii) a thiazole-substituted dioxetanone (TDO), a model used to study the firefly-luciferine bioluminescence.[20] The behavior of the position spread components at the CIs will be analyzed both for the ground and the excited state, furthermore its values will also be explored around a cut of the PES corresponding to the interpolation between the GS and the CI minima. In additional, the diagonalization of the $3 x 3$ tensor will also provide the principal components of both the TPS and the SPPS and their relationship with the CIs branching space will also be analyzed in detail.

Our contribution present a novel application of the position spread to the study of critical points for photophysical and photochemical properties such as the CIs. It will also be shown that its properties can provide an original way to locate CIs in complex PESs requiring only the solution of the GS wavefunction. Furthermore, an approximation of the branching space can be obtained by the simple knowledge of the SPPS.

\section{Methodology}

\subsection{The Total-Position Spread}

The Position Spread,[43] $\boldsymbol{\Lambda}$ is a tensor defined as the second moment cumulant of the Total-Position operator $\hat{\mathbf{R}}$ :

$$
\hat{\mathbf{R}}=\sum_{\mu} \hat{\mathbf{r}_{\mu}}
$$

where the sum runs over all the electron of the system. Then the cumulant is computed,

$$
\boldsymbol{\Lambda}=\left\langle\Psi\left|\hat{\mathbf{R}}^{2}\right| \Psi\right\rangle-\langle\Psi|\hat{\mathbf{R}}| \Psi\rangle^{2} .
$$

It is possible to partition the spread according to the electron spin: the position $\hat{\mathbf{r}}_{\mu}$ is written as the sum of the spin components $\hat{\mathbf{r}}_{\mu \alpha}+\hat{\mathbf{r}}_{\mu \beta}$, so that $\boldsymbol{\Lambda}$, which is quadratic in the position, becomes the sum of four terms. If the total spin projection vanishes, these terms are pairwise identical, in such a way that the final result is $\boldsymbol{\Lambda}=\boldsymbol{\Lambda}_{\alpha \alpha+\beta \beta}+\boldsymbol{\Lambda}_{\alpha \beta+\beta \alpha}$.

In the case of a wavefunction given by a single Slater determinant $\left|\Phi_{0}\right\rangle$, as is the case for the Hückel Hamiltonian, equation (4) becomes

$$
\begin{gathered}
\boldsymbol{\Lambda}=\left\langle\Phi_{0}\left|\hat{\mathbf{R}}^{2}\right| \Phi_{0}\right\rangle-\left\langle\Phi_{0}|\hat{\mathbf{R}}| \Phi_{0}\right\rangle^{2}= \\
=\sum_{I}\left\langle\Phi_{0}|\hat{\mathbf{R}}| \Phi_{I}\right\rangle\left\langle\Phi_{I}|\hat{\mathbf{R}}| \Phi_{0}\right\rangle-\left\langle\Phi_{0}|\hat{\mathbf{R}}| \Phi_{0}\right\rangle^{2},
\end{gathered}
$$

where the sum over $I$ is done over all the Slater determinants. Assuming an orthonormal orbital basis, and since the Total Position $\hat{\mathbf{R}}$ is a one-body 
operator, this sum can be restricted to $\mid \Phi_{0}>$ and the corresponding singlyexcited Slater determinants, $\left|\Phi_{i}^{k}\right\rangle$, only. Therefore the above equation reduces to

$$
\boldsymbol{\Lambda}=\sum_{i \in \mathrm{O}} \sum_{k \in \mathrm{V}}\left\langle\Phi_{0}|\hat{\mathbf{R}}| \Phi_{i}^{k}\right\rangle\left\langle\Phi_{i}^{k}|\hat{\mathbf{R}}| \Phi_{0}\right\rangle
$$

where $\mathrm{O}$ and $\mathrm{V}$ represents the sets of occupied and virtual spinorbitals, respectively. Finally, a given component of the TPS, xx for example, becomes

$$
\boldsymbol{\Lambda}_{x x}=\sum_{i \in \mathrm{O}} \sum_{k \in \mathrm{V}}\left\langle\phi_{i}\left|\hat{\mathbf{r}}_{x}\right| \phi_{k}\right\rangle\left\langle\phi_{k}\left|\hat{\mathbf{r}}_{x}\right| \phi_{i}\right\rangle
$$

Notice that a single excitation is necessarily either of type $\alpha$ or $\beta$, and this means that the different-spin components of a single Slater determinant necessarily vanish, $\boldsymbol{\Lambda}_{\alpha \beta+\beta \alpha}=0$. For this reason, the relative importance of $\boldsymbol{\Lambda}_{\alpha \beta+\beta \alpha}$ with respect to $\boldsymbol{\Lambda}_{\alpha \alpha+\beta \beta}$ is a powerful indicator of the Multi-Reference character of a wavefunction.

\subsection{The Spin Partitioned-Position Spread}

Since $\hat{\mathbf{R}}$ can be described the sum of two terms arising from the $\alpha$ and $\beta$ electrons, $\hat{\mathbf{R}}_{\alpha}$ and $\hat{\mathbf{R}}_{\beta}$, each term in Eq. 4 splits in four contributions, $\alpha \alpha, \beta \beta$, $\alpha \beta$ and $\beta \alpha$, so that:[44]

$$
\boldsymbol{\Lambda}=\boldsymbol{\Lambda}_{\alpha \alpha}+\boldsymbol{\Lambda}_{\beta \beta}+\boldsymbol{\Lambda}_{\alpha \beta}+\boldsymbol{\Lambda}_{\beta \alpha}
$$

where the different components of the TPS tensor are:

$$
\begin{aligned}
\boldsymbol{\Lambda}_{\alpha \alpha} & =\left\langle\Psi\left|\hat{\mathbf{R}}_{\alpha}^{2}\right| \Psi\right\rangle-\left\langle\Psi\left|\hat{\mathbf{R}}_{\alpha}\right| \Psi\right\rangle^{2} \\
\boldsymbol{\Lambda}_{\beta \beta} & =\left\langle\Psi\left|\hat{\mathbf{R}}_{\beta}^{2}\right| \Psi\right\rangle-\left\langle\Psi\left|\hat{\mathbf{R}}_{\beta}\right| \Psi\right\rangle^{2} \\
\boldsymbol{\Lambda}_{\alpha \beta} & =\left\langle\Psi\left|\hat{\mathbf{R}}_{\alpha} \hat{\mathbf{R}}_{\beta}\right| \Psi\right\rangle-\left\langle\Psi\left|\hat{\mathbf{R}}_{\alpha}\right| \Psi\right\rangle\left\langle\Psi\left|\hat{\mathbf{R}}_{\beta}\right| \Psi\right\rangle \\
\boldsymbol{\Lambda}_{\beta \alpha} & =\left\langle\Psi\left|\hat{\mathbf{R}}_{\beta} \hat{\mathbf{R}}_{\alpha}\right| \Psi\right\rangle-\left\langle\Psi\left|\hat{\mathbf{R}}_{\beta}\right| \Psi\right\rangle\left\langle\Psi\left|\hat{\mathbf{R}}_{\alpha}\right| \Psi\right\rangle
\end{aligned}
$$

Different symmetry relations occur among the spin-partitioned components of $\boldsymbol{\Lambda}$. Because $\hat{\mathbf{R}}_{\alpha}$ and $\hat{\mathbf{R}}_{\beta}$ commute, $\boldsymbol{\Lambda}_{\alpha \beta}$ and $\boldsymbol{\Lambda}_{\beta \alpha}$ components are equal. Moreover in the Heisenberg spin-chain there is no charge fluctuation among the sites, so that the spin-summed TPS is zero, while this does not hold in general for the spin-partitioned TPS which do not vanish, since the $\alpha$ and $\beta$ spins can fluctuate. As a consequence in this particular case, $\boldsymbol{\Lambda}_{\alpha \alpha}+\boldsymbol{\Lambda}_{\beta \beta}=-2 \boldsymbol{\Lambda}_{\alpha \beta}$. Finally, in this special case it occurs that $\boldsymbol{\Lambda}_{\alpha \alpha}$ and $\boldsymbol{\Lambda}_{\beta \beta}$ are also identical. This can be shown considering that:

$$
\left.\left.\left.\hat{\mathbf{R}}\right|^{m} \Psi(S)\right\rangle=\left.\sum_{i} C_{i}^{z}(S) \hat{\mathbf{R}}\right|^{m} \phi_{i}\right\rangle=0
$$


<smiles>c1ccc(/N=N/c2ccccc2)cc1</smiles>

b)

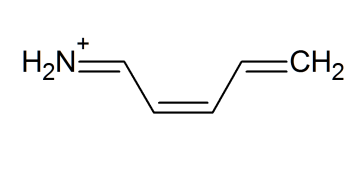

c)

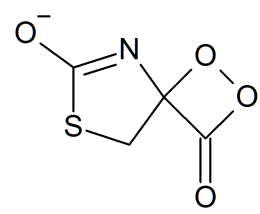

Fig. 1 Molecular formula of the compounds studied in the present contribution a) azobenzene (AB), b) PSB3, c) TDO.

because $\left.\left.\hat{\mathbf{R}}\right|^{m} \phi_{i}\right\rangle=0$ for any $\left.\left.\right|^{m} \phi_{i}\right\rangle$, where the wave function $\left.\left.\right|^{m} \Psi(S)\right\rangle$ is expressed as a linear combination of Slater determinants $\left.\left.\right|^{m} \phi_{i}\right\rangle$. It follows that:

$$
\left.\left.\left.\hat{\mathbf{R}}_{\alpha}\right|^{m} \Psi(S)\right\rangle=-\left.\hat{\mathbf{R}}_{\beta}\right|^{m} \Psi(S)\right\rangle
$$

from which

$$
\begin{aligned}
\left.\left.\hat{\mathbf{R}}_{\alpha}^{2}\right|^{m} \Psi(S)\right\rangle & \left.=-\left.\hat{\mathbf{R}}_{\alpha} \hat{\mathbf{R}}_{\beta}\right|^{m} \Psi(S)\right\rangle \\
\left.\left.\hat{\mathbf{R}}_{\beta} \hat{\mathbf{R}}_{\alpha}\right|^{m} \Psi(S)\right\rangle & \left.=-\left.\hat{\mathbf{R}}_{\beta}^{2}\right|^{m} \Psi(S)\right\rangle
\end{aligned}
$$

The equivalence $\boldsymbol{\Lambda}_{\alpha \alpha}=\boldsymbol{\Lambda}_{\beta \beta}$ is then easily proven. Therefore, in this investigation we will evaluate only one the spin-partinioned terms, namely $\boldsymbol{\Lambda}_{\alpha \alpha}$.

As a further simplification, regarding the translational invariance of the TPS, one can assume a coordinate system with $\left\langle\Psi\left|\hat{\mathbf{R}}_{\alpha}\right| \Psi\right\rangle=0$. This way the SP-TPS tensor can be written as:

$$
\boldsymbol{\Lambda}_{\alpha \alpha}=\left\langle\Psi\left|\hat{\mathbf{R}}_{\alpha}^{2}\right| \Psi\right\rangle
$$

\subsection{Computational Details}

The Franck-Condon geometries of the trans-AB, cis-PSB3 and TDO systems were optimized using the CASSCF method[45] as implemented in the MOLCAS 8 software package.[46] The choice of the active spaces was based on previous multiconfigurational studies and they will only be briefly described here.[12,9,47] For AB, we have choosen an active space distributing 10 electrons into 10 molecular orbitals [hereafter, CASSCF $(10,10)$ ], in particular including the most relevant $2 \pi$ and $2 \pi^{*}$ orbitals of each phenyl ring and all valence orbitals of the nitrogen atoms.[12] PSB3 was treated considering the full valence $\pi$ orbitals giving rise to a $\operatorname{CASSCF}(6,6) \cdot[9,10]$ Lastly, TDO was studied including the $\sigma$ and $\sigma^{*}$ orbitals of the $\mathrm{C}-\mathrm{C}$ and $\mathrm{O}-\mathrm{O}$ bonds of the dioxetanone ring, in conjunction with one of the non-bonding lone pair of one oxygen of the $\mathrm{O}-\mathrm{O}$ bond. In addition, the two most relevant $\pi$ and the most important $\pi^{*}$ orbitals centered in the $\mathrm{N}=\mathrm{C}_{-} \mathrm{O}^{-}$moiety of the thiazole heterocycle were also included, composing the final CASSCF(10,8) space.[47] Sketches of the active space orbitals are provided in Supplementary Information. 
The one-electron basis set of atomic natural orbital (ANO) type[48] with a $\mathrm{S}[4 \mathrm{~s} 3 \mathrm{p} 1 \mathrm{~d}] / \mathrm{C}, \mathrm{N}, \mathrm{O}[3 \mathrm{~s}, 2 \mathrm{p}, 1 \mathrm{~d}] / \mathrm{H}(2 \mathrm{~s} 1 \mathrm{p})$ contraction scheme (hereafter, ANO$\mathrm{S}-\mathrm{VDZP}$ ) was used for all multiconfigurational calculations. The minimum energy crossing points (MECPs) were optimized employing the same active space used for the ground-state minimizations and starting from the geometries previously reported in the literature.[12,9,47] A balanced description of the ground and excited states was achieved by computing two states in the CASSCF method with equal weight (state-average CASSCF procedure). The energy degeneracy between the $S_{1}$ and the $S_{0}$ states was imposed by means of the restricted Lagrange multipliers technique, in which the lowest-energy point within the restriction of degeneracy between the two considered states is obtained.[49] The linear interpolation of internal coordinates (LIIC) method was employed to connect both Franck-Condon and MECP structures. The resulting photochemical paths are thus not optimized even though they are extremely useful to study the behavior of the TPS and SPPS tensors at the $S_{1} / S_{0}$ energy degeneracy areas considered in this work.

The TPS and SPPS quantities were computed at the CASSCF level[42] employing the same active spaces described above and making use of their implementation in the MOLPRO software package.[50] Throughout all the manuscript we report the values obtained after the diagonalization of the full tensor. Note that separate diagonalizations are performed for TPS and SPPS tensors, however the obtained eigenvectors, i.e. the principal direction, do not differ significantly.

\section{Results}

\subsection{Description of the CIs minima}

The GS and CIs optimized geometries are sketched in Figure 2, while the cartesian coordinates are given in Supplementary Information. Note that the most relevant internal coordinates driving the relaxation to the CI region for all the systems under study are also given in Figure 2.

The isomerization of the $\mathrm{AB}$ switch represent a classical case study in photochemistry and a large quantity of computational studies have been devoted to the resolution of its mechanism, $[12,13]$ both in terms of the CI structural characterization and terms of the resolution of the lifetime and quantum yields of the isomerization process. In our case one may unsurprisingly note that the GS minimum shows a perfectly planar structure, whereas in the case of the CI the dihedral $(\Phi)$ angle decreases to 90 degrees hence giving a quasi perpendicular arrangement of the two phenyl group. Such an evolution is coherent with previous findings reported in the literature and is also perfectly justified by the fact that the CI drives the trans/cis isomerization process proceeding via the weakening of the azo $\pi$ double bond, hence allowing the free rotation around the dihedral. Note however that, once again coherently with previous 
a)

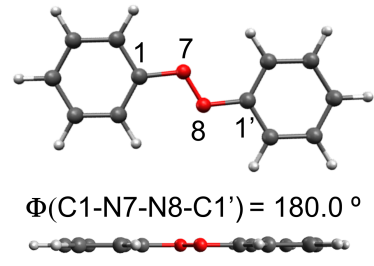

d)

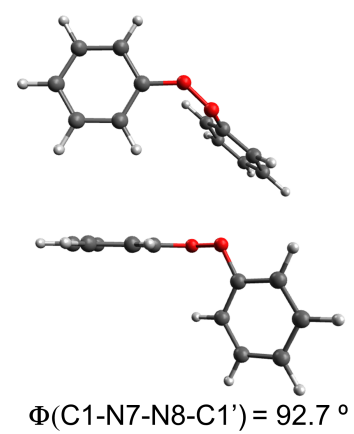

b)

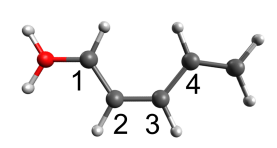

$\theta(\mathrm{C} 1-\mathrm{C} 2-\mathrm{C} 3-\mathrm{C} 4)=0.1^{\circ}$

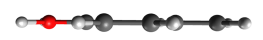

e)
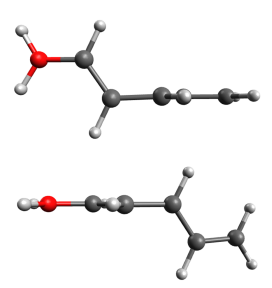

$\theta(C 1-C 2-C 3-C 4)=-92.5^{\circ}$ c)
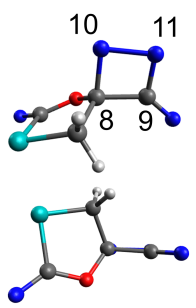

$\mathbf{R}(010-011)=1.57 \AA$

f)
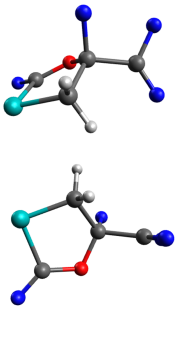

Fig. 2 Optimized geometries for the GS (top panel) and the CI (bottom panel) of AB a) and d), PSB3 b) and e) and TDO c) and f). The values of the most important coordinates driving the relaxation to the CI are also reported for the three systems.

studies, $[12,13]$ the rotation around the dihedral is also accompanied by the pyramidalization of the $\mathrm{N} 8$ atom.

A similar process and a similar nature of the CI is also observed in the case of the PSB3 system whose photochemistry is dominated by the cis/trans isomerization around the central carbon double bond.[9,10] The interest of PSB3 resides in the fact that it can be considered as one of the simplest model for the retinal photo-isomerization, i.e. a photochemical reaction that triggers the vision process in superior animals and that may be used for signaling in bacteria. As such, PSB3 has been largely studied also using highly-correlated methods like equation of motion coupled-cluster. $[9,10]$ Once again, from a planar structure in the GS the system evolves to a CI in which the two sub-units are oriented perpendicularly as witnessed by the evolution of the $\theta$ dihedral from 0 to 90 degrees. However, as it was the case for $\mathrm{AB}$ the rotation is accompanied by a slight pyrimidalization of the $\mathrm{C} 3$ atom and by a change in the bond length alternation (BLA) that witnesses the weakening of the carbon-carbon double bond.

Finally, in the case of the TDO molecule the CI region is associated with the enlargement of the oxygen-oxygen bond leading to the formal breaking of the four-membered ring.[47] Interestingly, the dioxetane bond breaking is also responsible of the chemiluminescent properties of the system since the 
thermally activated bond cleavage will result in the population of excited states that can induce luminescence.

It is therefore clear that the protocol used in the present study allows to correctly reproduce the main recognized structural features of the CIs of the three chosen systems. Even though in the present study we are not interested in the quantitative exploration of the PES to precisely position the CI region, such considerations validate the present protocol assuring its robustness.

\subsection{TPS and SPPS behavior at the CI region}

Before discussing in details the values obtained in the case of the three selected compounds, we resort to a simplified model still presenting a CI, i.e. the ethylene molecule. More in details, we study the behavior of the $\Lambda$ tensor along the torsion of the $\mathrm{H}-\mathrm{C}-\mathrm{C}-\mathrm{H}$ dihedral angle defining the planarity of the system.

The results are plotted in Figure 3 and they clearly show that at the CI point the TPS curves for $S_{0}$ and $S_{1}$ crosses abruptly producing a discontinuity in correspondence of the CI seam. This result is not surprising if one consider the diabatic representation of the electronic states. One can consider that since the two surfaces exchange their nature, hence the properties of each state must switch as well. It is important to note however that the behavior of the TPS is strongly different in the case of an avoided crossing [40,41], when the change takes place smoothly showing a $\Lambda$ maximum value. In contrast, the CI region does not evidence a clear maximum and the TPS curves present an evident discontinuity. This different behavior can be related to the different nature of the electronic states in the two cases: indeed avoided crossings can be constructed via a smooth linear combination of the involved diabatic states leading to their adiabatic mixing, and hence to the increase in electron delocalization. CIs are characterized by energy degeneracy and hence by the sudden increase in the mixing between the states at the CI seam and to a strong non-adiabatic nature. However, it should be underlined that the discontinuity in the localization tensor will appear only in the case of the crossing of states showing a different nature, for instance neutral and ionic ones, and is hence not a totally general properties of every conical intersection.

The results of both the TPS $\left(\Lambda^{T P S}\right)$ and of SPPS for same spin $\left(\Lambda_{\alpha \alpha \beta \beta}^{S P P S}\right)$ and different spin $\left(\Lambda_{\alpha \beta \alpha \beta}^{S P P S}\right)$ components are collected in Table 1 . Note that we report here the principal components values of $\Lambda$ obtained after diagonalization of the calculated tensor, indeed and because of the lack of symmetry of the optimized CI non diagonal components $\left(\Lambda_{X} Y, \Lambda_{X} Z, \Lambda_{Y} Z\right)$ are in general different from zero. Some general trends confirming what obtained for the simpler ethylene model can be underlined by analyzing the behavior of the TPS. Indeed, in all the cases a quasi-degeneracy for the value of $\Lambda^{T P S}$ is observed between $S_{0}$ and $S_{1}$. The small deviations observed being mostly due to numerical instability in the optimization of the CIs geometry that slightly break the energy degeneracy. Remarkably enough, the degeneracy is 

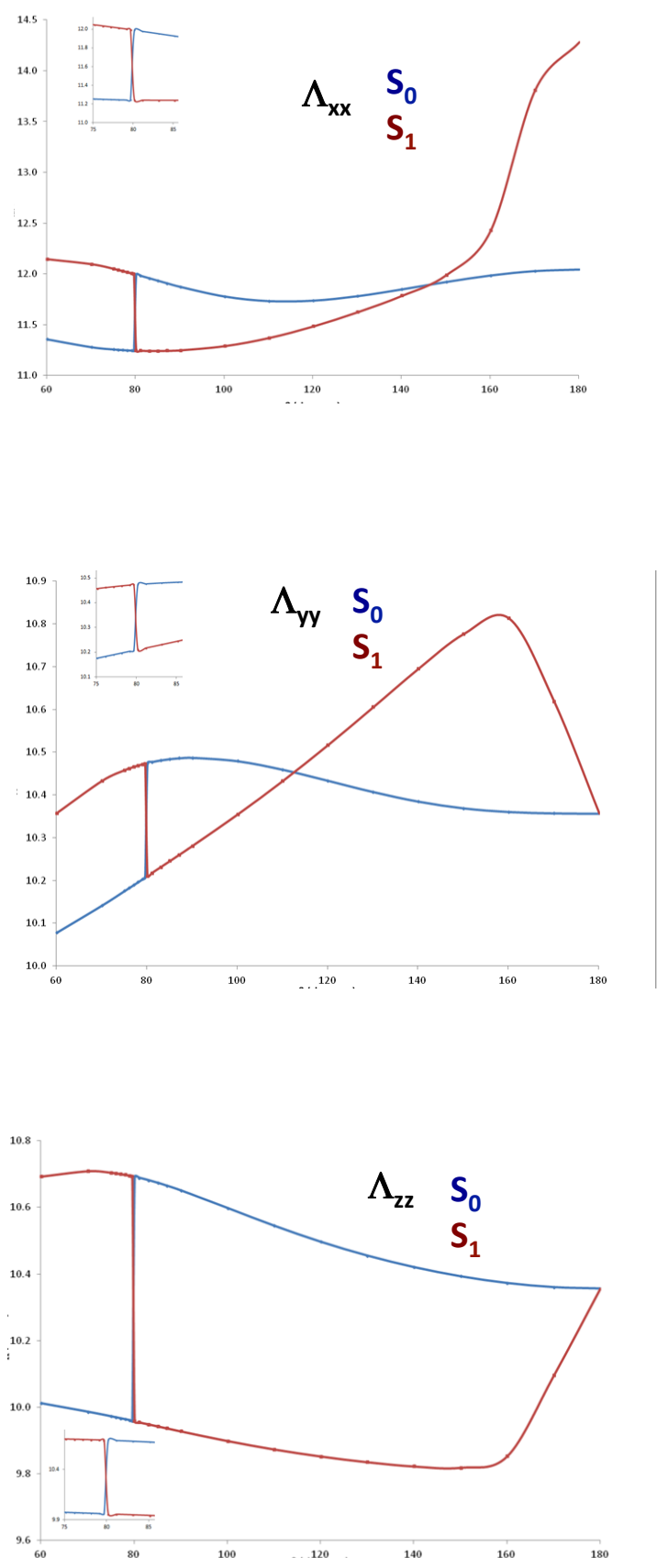

Fig. 3 Values of the TPS $\Lambda$ along the principal coordinate $x x, y y, z z$ calculated along the torsion of the dihedral angle of ethylene for the ground and the first excited state. Note the crossing of the values of $\Lambda$ for all the three principal components, the CIs region is also evidenced in the inlay zoom. 
Table 1 Values of the TPS and SPPS tensor $\Lambda$ in $a . u .^{2}$ calculated at the optimized CI geometry for azobenzene, PSB3, and TDO.

\begin{tabular}{|c|c|c|c|c|c|c|c|c|c|c|}
\hline & & & & Azobenzene & & & TDO & & & PSB3 \\
\hline & & $X X$ & $Y Y$ & $Z Z$ & $X X$ & $Y Y$ & $Z Z$ & $X X$ & $Y Y$ & $Z Z$ \\
\hline \multirow[t]{2}{*}{$\Lambda^{T P S}$} & $S_{0}$ & 52.59 & 53.14 & 61.27 & 36.08 & 37.54 & 38.88 & 23.06 & 23.51 & 27.44 \\
\hline & $S_{1}$ & 52.94 & 53.15 & 62.99 & 35.89 & 37.46 & 38.73 & 22.74 & 23.33 & 26.75 \\
\hline \multirow{2}{*}{$\Lambda_{\alpha \alpha \beta \beta}^{S P P S}$} & $S_{0}$ & 54.13 & 55.56 & 74.65 & 37.56 & 38.32 & 47.36 & 23.36 & 23.77 & 29.49 \\
\hline & $S_{1}$ & 54.15 & 54.92 & 65.65 & 37.8 & 38.37 & 48.17 & 23.28 & 23.51 & 45.55 \\
\hline \multirow{2}{*}{$\Lambda_{\alpha \beta \alpha \beta}^{S P P S}$} & $S_{0}$ & -13.56 & -2.44 & -1.33 & -9.94 & -0.45 & -0.35 & -2.35 & -0.24 & -0.01 \\
\hline & $S_{1}$ & -2.73 & -1.76 & -1.15 & -11.21 & -0.67 & -0.38 & -18.91 & -0.60 & -0.01 \\
\hline
\end{tabular}

spread over all the three principal components of the tensor, and once again it can be related to the fact that at the CI region the diabatic states will give raise to a non-adiabatic exchange. As it was proven in the case of ethylene torsion such a phenomenon should be related to the insurgence of discontinuity in the TPS value at the CI seam. It is however remarkable that indeed no significant difference in the value of $\Lambda^{T P S}$ can be observed among the principal components, that are on the contrary almost quasi-degenerate.

The general tendencies shown by the two smallest principal components are however interesting and indeed deserve a proper discussion. If, for the sake of simplicity we consider $\mathrm{AB}$ in its ground state, we clearly see that along the interpolation bridging the Franck-Condon and the CI region, the value of $\Lambda_{X X}$ goes from 48 to $53 a u^{2}$, while $\Lambda_{Y Y}$ varies between 58 and $53 a u^{2}$. This behavior is actually the same for the $S_{1}$ state. Such a phenomenon can be easily interpreted taking into account a very simple model based on the different orbital occupation between the electronic states and its coupling with the nuclear coordinates. Indeed, and again considering AB, at the Franck-Condon region one can state that $S_{0}$ state will show an almost double occupation of the highest $\pi$ orbital, while $S_{1}$ will be characterized by almost single occupied $\pi$ and $\pi *$ orbitals. Hence, the progress towards the conical intersection will be characterized by the decrease in the occupation of the $\pi$ orbitals and the corresponding increase of the $\pi *$ weight, due to the mixing between the ground and excited determinants. Because of the different symmetry of the orbitals this aspect is reflected in the different and symmetric behavior of the $\Lambda_{X X}$ and $\Lambda_{Y Y}$ components. The $S_{1}$ state, on the other hand, will experience the exact opposite transition, and will also undergo a similar mixing. Hence, while approaching the CI the weight of the $\pi$ orbital should be increased at the expense of the $\pi *$ one. Hence the behavior of the two TPS components should be similar to the one of the $S_{0}$ state, as confirmed by our calculations.

However, the behavior of the SPPS is more interesting, even though no clear trends can be extracted from the same spin component $\Lambda_{\alpha \alpha \beta \beta}^{S P P S}$ whose behavior is similar to that of the TPS, although the degeneracy between the ground and the excited states is partially lost. On the contrary, the different 
spin component $\Lambda_{\alpha \beta \alpha \beta}^{S P P S}$ deserves much more attention. Firstly note that the different spin partition has negative values for all the three principal components. This is not surprising since while the TPS and the same spin partition are second-order cumulant and hence positive definite, $\Lambda_{\alpha \beta \alpha \beta}^{S P P S}$ is a joint cumulant whose sign is not defined. Furthermore, and differently from the TPS and the same spin partition, only one component of $\Lambda_{\alpha \beta \alpha \beta}^{S P P S}$ is significantly different from zero at the CI seams. This behavior can be rationalized considering that $\Lambda_{\alpha \beta \alpha \beta}^{S P P S}$ is strictly zero for single-determinant wavefunctions and assumes high values only in the case of strongly correlated systems.[44] If we consider a CI we should remind that two coordinates exists that break the degeneracy between the states, obviously, as a consequence of the energy degeneracy lifting, the multiconfigurational nature of the wavefunction will also be reduced. Therefore, we can infer that the two close to zero components of $\Lambda_{\alpha \beta \alpha \beta}^{S P P S}$ should be related to the CI branching space, or the $\mathrm{g}$-h plane, that could be approximated by the corresponding eigenvectors obtained during the diagonalization procedure. On the contrary the third component, significantly different from zero, should represent the CI surface in which the energy degeneracy, and, hence, the high static correlation are maintained. Note that this general behavior is well reproduced for both the ground and the excited states, although the magnitude of the effect is not exactly the same for the two states, once again most probably due to the numerical uncertainty in the optimization procedure, leading to a non-perfect energy degeneracy.

\subsection{Coordinate interpolation}

The behavior of TPS and SPPS tensors along the LIIC coordinates connecting the Franck-Condon and the CI regions is represented in Figure 4 for AB, PSB3, and TDO. The TPS behavior points to the fact that the reaching the CI is accompanied by a degeneracy between $S_{0}$ and $S_{1}$ that will ultimately lead to the discontinuity observed in the case of ethylene.

On the other hand, the SPPS values along the progression toward the CI structures confirm that while two components stay close to zero, the absolute value of the third one increase significantly, both for the ground and the first excited state. Note however that the behavior is different among the three molecular systems, in particular for the excited state. Indeed, while in the case of AB both the $S_{0}$ and $S_{1}$ dominant component of the SPPS decrease smoothly as the system approaches the CI regions, the $S_{1}$ dominant component of PSB3 presents a maximum, hence approaching back to zero, to again diminishes its value while further approaching the CI. However, the fact that upon diagonalization only one principal component differing significantly from zero is confirmed. 
Total Position Spread Tensor

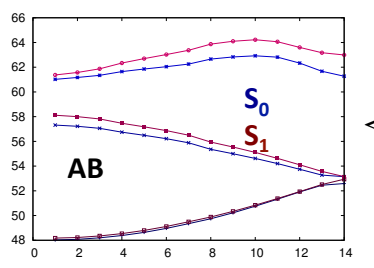

LIIC

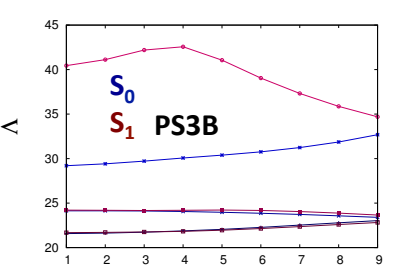

LIIC

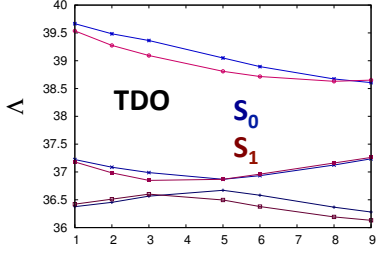

LIIC

Spin Partitioned Position Spread Tensor

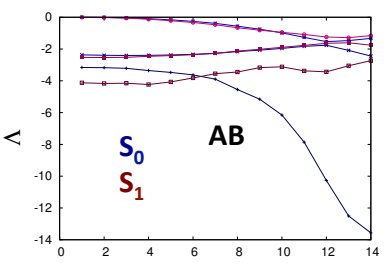

LIIC

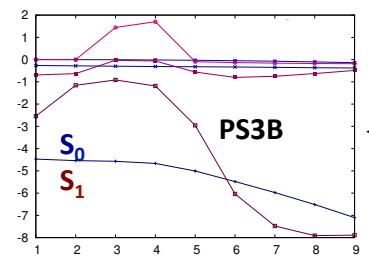

LIIC

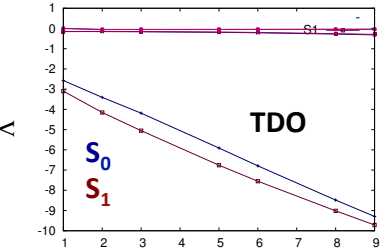

IIIC

Fig. 4 Values $\left(a . u .^{2}\right.$ of the three $(X X, Y Y, Z Z)$ principal components of the TPS (top panel) and SPPS (bottom panel) tensor for the $S_{0}$ and $S_{1}$ state calculated along the LIIC bridging the Franck-Condon and the CI region for AB, PSB3, and TDO.

\section{Conclusions}

In this contribution we have for the first time applied the concept of the position spread tensor to the study of conical intersections in photoactive molecular systems. In particular we have shown that in presence of a conical intersection the TPS shows a clear discontinuity related to the sudden exchange between the $S_{0}$ and $S_{1}$ nature, that is also accompanied by a degeneracy of the TPS value at the CI seam. Most remarkably, this behavior is different from what was previously observed for avoided crossings, in which the TPS presents a maximum. Hence, following the TPS discontinuity in the ground state could provide an alternative way to place the CI only requiring the knowledge of the GS properties. However the discontinuity will be rigorously observed only in the case of the crossing of states having a different nature.

In the case of the SPPS, one may notice that at the CI seam two components have values close to zero while only the third one differs significantly. Since SPPS may be related to the amount of electron correlation, and in par- 
ticular is supposed to vanish for single-determinant wavefunctions, we can infer that the two smallest components may represent the degeneracy-lifting coordinates, i.e. the branching plane, since the electron correlation is supposed to diminish along this space.

In the future we plan to extend this study by the explicit calculation of the branching space and provide its relation with the SPPS principal components. At the same time, we plan to enlarge the study to the investigation of the behavior of singlet-triplet crossing points for which the spin partition can unravel even more important features.

\section{Acknowledgments}

Support from the Université de Lorraine and Université Toulouse III as well as from French CNRS is gratefully acknowledged. A.F.-M. also thanks the Grand Est regional government (France) for financial support.

This work is dedicated to the memory of Janos G. Angyán who was an inspiring scientist as well as a good colleague and friend, the memory of his scientific and human qualities will not be forgotten. The authors are also indebted to many fruitful discussion with Janos on the subject of this paper.

\section{References}

1. D.R. Yarkony, Chemical Reviews 112(1), 481 (2012)

2. T. Yonehara, K. Hanasaki, K. Takatsuka, Chemical Reviews 112(1), 499 (2012)

3. I. Schapiro, F. Melaccio, E.N. Laricheva, M. Olivucci, Photochem. Photobiol. Sci. 10(6), 867 (2011)

4. J.P. Malhado, M.J. Bearpark, J.T. Hynes, Frontiers in Chemistry 2, 97 (2014)

5. F. Bernardi, M. Olivucci, M.A. Robb, Chem. Soc. Rev. 25(5), 321 (1996)

6. D.R. Yarkony, The Journal of Physical Chemistry A 105(26), 6277 (2001)

7. R. Improta, F. Santoro, L. Blancafort, Chemical Reviews 116, 3540 (2016)

8. A. Giussani, J. Segarra-Martí, D. Roca-Sanjuán, M. Merchán, Topics in Current Chemistry 355, 57 (2015)

9. S. Gozem, A.I. Krylov, M. Olivucci, Journal of Chemical Theory and Computation 9(1), $284(2013)$

10. S. Gozem, A.I. Krylov, M. Olivucci, Journal of Chemical Theory and Computation 9(1), $284(2013)$

11. Chem. Sci. 9(20), 4671 (2018)

12. A. Cembran, F. Bernardi, M. Garavelli, L. Gagliardi, G. Orlandi, Journal of the American Chemical Society 126(10), 3234 (2004)

13. S. Gozem, F. Melaccio, R. Lindh, A.I. Krylov, A.A. Granovsky, C. Angeli, M. Olivucci, Journal of Chemical Theory and Computation 9(10), 4495 (2013)

14. L.A. Estrada, A. Frances-Monerris, I. Schapiro, M. Olivucci, D. Roca-Sanjuan, Phys. Chem. Chem. Phys. 18(48), 32786 (2016)

15. L. Martinez-Fernandez, A.J. Pepino, J. Segarra-Marti, J. Jovaisaite, I. Vaya, A. Nenov, D. Markovitsi, T. Gustavsson, A. Banyasz, M. Garavelli, R. Improta, JOURNAL OF THE AMERICAN CHEMICAL SOCIETY 139(23), 7780 (2017)

16. M.G.S. Londesborough, J. Dolanský, L. Cerdán, K. Lang, T. Jelínek, J.M. Oliva, D. Hnyk, D. Roca-Sanjuán, A. Francés-Monerris, J. Martinčík, M. Nikl, J.D. Kennedy, Advanced Optical Materials 5(6), 1600694 (2017)

17. M.G.S. Londesborough, D. Hnyk, J. Bould, L. Serrano-Andres, V. Sauri, J.M. Oliva, P. Kubat, T. Polivka, K. Lang, Inorganic Chemistry 51(3), 1471 (2012) 
18. A. Giussani, M. Merchán, D. Roca-Sanjuán, R. Lindh, Journal of Chemical Theory and Computation $\mathbf{7}(12), 4088$ (2011)

19. M. Vacher, I. Fdez. Galván, B.W. Ding, S. Schramm, R. Berraud-Pache, P. Naumov, N. Ferré, Y.J. Liu, I. Navizet, D. Roca-Sanjuán, W.J. Baader, R. Lindh, Chemical Reviews $\mathbf{0}(0)$, null

20. N. Isabelle, L. Ya-Jun, F. Nicolas, R.S. Daniel, L. Roland, ChemPhysChem 12(17), 3064

21. E. Teller, The Journal of Physical Chemistry 41(1), 109 (1937)

22. G. Herzberg, H.C. Longuet-Higgins, Discuss. Faraday Soc. 35(0), 77 (1963)

23. I. Fdez. Galván, M.G. Delcey, T.B. Pedersen, F. Aquilante, R. Lindh, Journal of Chemical Theory and Computation 12(8), 3636 (2016)

24. X. Zhang, J.M. Herbert, The Journal of Chemical Physics 141(6), 064104 (2014)

25. S. Fatehi, E. Alguire, Y. Shao, J.E. Subotnik, The Journal of Chemical Physics 135(23), $234105(2011)$

26. A. Tajti, P.G. Szalay, The Journal of Chemical Physics 131(12), 124104 (2009)

27. H. Lischka, M. Dallos, P.G. Szalay, D.R. Yarkony, R. Shepard, The Journal of Chemical Physics 120(16), 7322 (2004)

28. K.L. Bak, P. Jo/rgensen, H.J.A. Jensen, J. Olsen, T. Helgaker, The Journal of Chemical Physics 97(10), 7573 (1992)

29. B.H. Lengsfield, P. Saxe, D.R. Yarkony, The Journal of Chemical Physics 81(10), 4549 (1984)

30. V. Vetere, A. Monari, G.L. Bendazzoli, S. Evangelisti, B. Paulus, The Journal of Chemical Physics 128(2), 024701 (2008)

31. A. Monari, G.L. Bendazzoli, S. Evangelisti, The Journal of Chemical Physics 129(13), 134104 (2008)

32. B.G. Luigi, E. Stefano, M. Antonio, International Journal of Quantum Chemistry 111(13), 3416

33. G.L. Bendazzoli, S. Evangelisti, A. Monari, B. Paulus, V. Vetere, Journal of Physics: Conference Series 117(1), 012005 (2008)

34. E. Giner, G.L. Bendazzoli, S. Evangelisti, A. Monari, The Journal of Chemical Physics 138(7), 074315 (2013)

35. S. Evangelisti, G.L. Bendazzoli, A. Monari, Theoretical Chemistry Accounts 126(3), $257(2010)$

36. R. Resta, Phys. Rev. Lett. 80, 1800 (1998)

37. R. Resta, S. Sorella, Phys. Rev. Lett. 82, 370 (1999)

38. R. Resta, Phys. Rev. Lett. 95, 196805 (2005)

39. R. Resta, The Journal of Chemical Physics 124(10), 104104 (2006)

40. O. Brea, M. El Khatib, C. Angeli, G.L. Bendazzoli, S. Evangelisti, T. Leininger, Journal of Chemical Theory and Computation 9(12), 5286 (2013)

41. O. Brea, M. El Khatib, G.L. Bendazzoli, S. Evangelisti, T. Leininger, C. Angeli, The Journal of Physical Chemistry A 120(27), 5230 (2016)

42. M.E. Khatib, T. Leininger, G.L. Bendazzoli, S. Evangelisti, Chemical Physics Letters 591, 58 (2014)

43. B.G. Luigi, E.K. Muammar, E. Stefano, L. Thierry, Journal of Computational Chemistry $\mathbf{3 5}(10), 802$

44. M. El Khatib, O. Brea, E. Fertitta, G.L. Bendazzoli, S. Evangelisti, T. Leininger, The Journal of Chemical Physics $142(9), 094113$ (2015)

45. B.O. Roos, P.R. Taylor, P.E.M. Siegbahn, Chemical Physics 48, 157 (1980)

46. F. Aquilante, J. Autschbach, R.K. Carlson, L.F. Chibotaru, M.G. Delcey, L. De Vico, I. Fdez. Galván, N. Ferré, L.M. Frutos, L. Gagliardi, M. Garavelli, A. Giussani, C.E. Hoyer, G. Li Manni, H. Lischka, D. Ma, P.A. Malmqvist, T. Müller, A. Nenov, M. Olivucci, T.B. Pedersen, D. Peng, F. Plasser, B. Pritchard, M. Reiher, I. Rivalta, I. Schapiro, J. Segarra-Martí, M. Stenrup, D.G. Truhlar, L. Ungur, A. Valentini, S. Vancoillie, V. Veryazov, V.P. Vysotskiy, O. Weingart, F. Zapata, R. Lindh, Journal of Computational Chemistry 37, 506 (2015)

47. F. Liu, Y. Liu, L.D. Vico, R. Lindh, Chemical Physics Letters 484(1), 69 (2009)

48. K. Pierloot, B. Dumez, P.O. Widmark, B.O. Roos, Theoretica Chimica Acta 90(2-3), 87 (1995) 
49. L. De Vico, M. Olivucci, R. Lindh, Journal of Chemical Theory and Computation 1(5), $1029(2005)$

50. H.J. Werner, P.J. Knowles, G. Knizia, F.R. Manby, M. Schütz, P. Celani, W. Györffy, D. Kats, T. Korona, R. Lindh, A. Mitrushenkov, G. Rauhut, K.R. Shamasundar, T.B. Adler, R.D. Amos, A. Bernhardsson, A. Berning, D.L. Cooper, M.J.O. Deegan, A.J. Dobbyn, F. Eckert, E. Goll, C. Hampel, A. Hesselmann, G. Hetzer, T. Hrenar, G. Jansen, C. Köppl, Y. Liu, A.W. Lloyd, R.A. Mata, A.J. May, S.J. McNicholas, W. Meyer, M.E. Mura, A. Nicklass, D.P. O'Neill, P. Palmieri, D. Peng, K. Pflüger, R. Pitzer, M. Reiher, T. Shiozaki, H. Stoll, A.J. Stone, R. Tarroni, T. Thorsteinsson, M. Wang. Molpro, version 2015.1, a package of ab initio programs (2015). See 